\title{
Fusion of Multispectral and Panchromatic Images based on Fourier Filtering in HSV Color Space
}

\author{
Meenu Manchanda \\ Associate Professor, Vaish College of Engineering \\ Rohtak, Haryana, India
}

\section{ABSTRACT}

A large number of imaging sensors in the field of remote sensing capture images with different spatial and spectral resolutions. Given a low spatial resolution multispectral image(MS) and a high spatial resolution panchromatic image (PAN), the proposed algorithm aims to enhance the spatial resolution of MS images while simultaneously preserving the high spectral information contained in it. Thus, a method of fusion of MS and PAN images based on Fourier filtering in HSV color space is proposed. Experimental results prove that the proposed method produces better fusion results.

\section{INTRODUCTION}

Image fusion has emerged as an advanced and promising research area with the aim of combining information from multiple input images into a single fused image [8], [9]. An increase in the availability of space borne imaging sensors in the field of remotesensing [5], [6] provided strong motivations. Remote sensing images are characterized by their spectral and spatail resolutions. Spatial resolution relates to the bandwidth and the sampling rate over which the sensor captures information about the scene whereas spatial resolution relates to the smallest features in the scene that can be resolved. Thus due to system tradeoffs, these sensors tend to capture either high spectral resolution but low spatial resolution multispectral (MS) images or low spectral resolution but high spatial resolution panchromatic (PAN) images. However there are many applications such as mapping of land use, precision agriculture and classification of rural/urban areas that demands a high spatial as well as high spectral resolution image. This requirement of a single image with high spatial and spectral resolution led to the fusion of MS and PAN images [3]. Thus in the field of remote sensing, image fusion is the process of merging two images of different spatial and spectral resolutions in order to produce a single fused image that has highest spatial resolution while preserving high spectral resolution. Fusion algorithms based on injecting high frequency information from PAN image into MS image have proved to be superior in enhancing the spatialresolution of MS images with minimum distortion of spectral resolution. Fourier transform (FT) has emerged as an important tool in image processing [1], [4], [7]. The high-frequency (HF) component of FT is highly correlated with the spatial texture information contained in the original image and the low frequency (LF) component is correlated with the gray information. Therefore, the HF component of PAN image can be fused with the LF component of MS image to produce a fused image with high spatial resolution as well as with high spectral information. Thus in order to synthesize MS images with a high spatial resolution, this paper proposes fast Fourier transform (FFT) based image fusion algorithm in HSV (huesaturation-value) color space. The rest of the paper is structured as follows: Section 2 provides detail on FFT, Section 3 describes the proposed fusion algorithm, Section 4 presents results and discussion and finally Section 5 draws the conclusion.

\section{FAST FOURIER TRANSFORM BASED FUSION}

(FFT)

The 2D discrete Fourier transform (DFT) of an image $x\left(n_{1}, n_{2}\right)$ of size $M \times N$ is defined as:

$X\left(k_{1}, k_{2}\right)=\frac{1}{M N} \sum_{n_{1}=0}^{M-1} \sum_{n_{2}=0}^{N} x\left(n_{1}, n_{2}\right) e^{-j 2 \pi\left(\frac{k_{1}}{M} n_{1}+\frac{k_{2}}{N} n_{2}\right)}$

where $0 \leq k_{1} \leq M-1$ and $0 \leq k_{2} \leq N-1$. Similarly, 2D inverse-DFT is defined as:

$$
x\left(n_{1}, n_{2}\right)=\sum_{k_{1}=0}^{M-1} \sum_{k_{2}=0}^{N} x\left(k_{1}, k_{2}\right) e^{j 2 \pi\left(\frac{k_{1}}{M} n_{1}+\frac{k_{2}}{N} n_{2}\right)}
$$

where $0 \leq n_{1} \leq M-1$ and $0 \leq n_{2} \leq N-1$. Both DFT and inverse-DFT are separable transformations.

$$
X\left(k_{1}, k_{2}\right)=\frac{1}{M N} \sum_{n_{1}=0}^{M-1}\left[\sum_{n_{2}=0}^{N} x\left(n_{1}, n_{2}\right) e^{-j 2 \pi \frac{k_{2}}{N} n_{2}}\right] e^{-j 2 \pi \frac{k_{1}}{M} n_{1}}
$$


Fast Fourier transform (FFT) is an efficient way to compute DFT. It exploits two important properties, symmetry and periodicity, of the phase factor i.e. $e^{\frac{-\jmath^{2} \pi}{N}}$ to compute DFT.

1) Symmetry property:

$$
e^{\frac{-j 2 \pi\left(k_{1}+\frac{M}{2}\right)}{M}}=-e^{\frac{-j 2 \pi k_{1}}{M}}
$$

and

$$
e^{\frac{-j 2 \pi\left(k_{2}+\frac{N}{2}\right)}{N}}=-e^{\frac{-j 2 \pi k_{2}}{N}}
$$

2) Periodicity property:

$$
e^{\frac{-j 2 \pi\left(k_{1}+M\right)}{M}}=e^{\frac{-j 2 \pi k_{1}}{M}}
$$

and

$$
e^{\frac{-j 2 \pi\left(k_{2}+N\right)}{N}}=e^{\frac{-j 2 \pi k_{2}}{N}}
$$

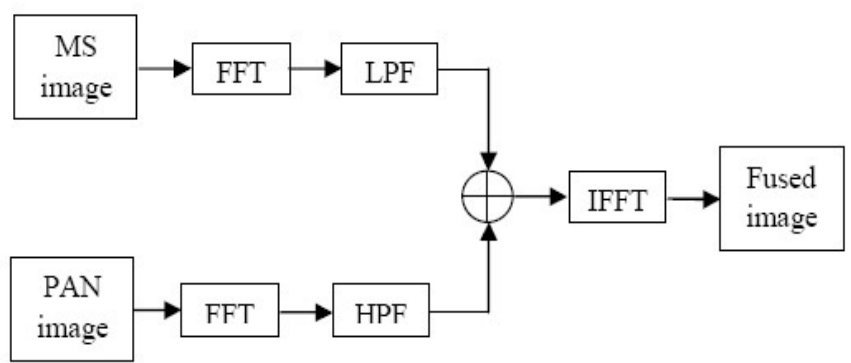

Fig 1: Block diagram showing FFT based image fusion

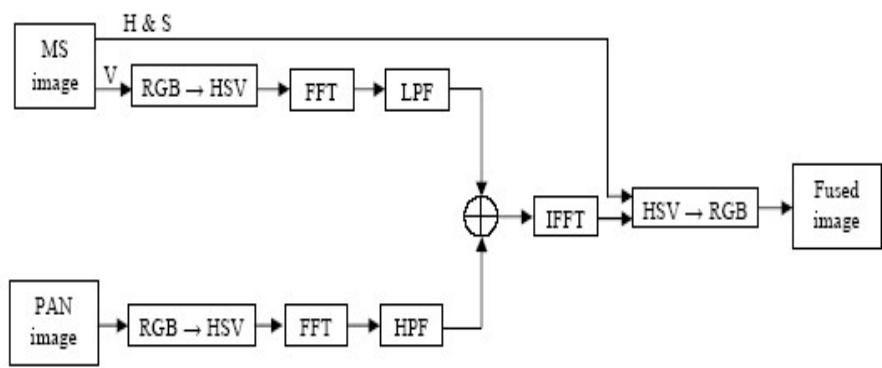

Fig 2: FFT- HSV based fusion

\section{PROPOSED WORK}

The main aim of the proposed work is to fuse two images, a PAN image and a MS image that have been captured at different spatial resolution to produce a single image that contains spatial information of high spatial resolution image i.e. PAN image and spectral information of low spatial resolution image i.e. MS image. The proposed work is illustrated in Fig. 1 and is described as follows:

1) Input MS and PAN images are first converted from RGB (red-green-blue) color space to HSV (huesaturationvalue) color space.

2) The PAN image and the V-channel information of the MS image are transformed into frequency domain using 2D-FFT.

3) The fast Fourier transformed V-channel information of MS image is then passed through an appropriate LPF whereas the fast Fourier transformed PAN image is

passed through an appropriate HPF.

4) The low-pass filtered information is then added to the high-pass filtered information.

5) The sum information is transformed back into the spatial domain using 2D-IFFT to produce V-channel information i.e. Vfused of the fused image.

6) The fused V-channel information along with the color information (i.e. $\mathrm{H}$ and $\mathrm{S}$ ) of the multispectral image is finally converted from HSV color space to RGB color space to produce spatially enhanced MS image.

\section{RESULTS AND DISCUSSIONS}

The proposed method is experimented on different sets of MS and PAN images of size 256256. MS images with low spatial resolution but high spectral resolution are shown in Fig.2 (a) - Fig. 4(a) and PAN images with high spatial resolution but low spectral resolution is shown in Fig. 2(b) - Fig. 4(b) respectively. The fused images obtained using the proposed method is shown in Fig. 2(d) - Fig. 4(d) respectively. The fused images obtained using FFT based fusion method is also shown in Fig. 2(c) - Fig. 4(c) respectively. From these fused images it is observed that the proposed method produces the fused images with improved spatial resolution and well preserved spectral information in comparison to the FFT based fusion method. This is because fusion in RGB color space does not utilize the correlation between different image channels [2] and generates undesirable visual effects in the fusion results whereas HSV color is much closer to humans' perception to Color and therefore fusion in HSV color space yields better fusion results. 


\section{REFERENCES}

[1] M Ehlers and D Tomowski. On segment based image fusion. Object-Based Image Analysis, pages 735-754, 2008.

[2] L. Georgieva, T. Dimitrova, and N. Angelov. Rgb and hsv colour models in colour identification of digital traumas images. Citeseer.

[3] L. Hu, B. Xiangli, L. Su, and Y. Yuan. Fusion of multispectral and panchromatic images using lab transform based on fourier filtering. In 2012 IEEE International Geoscience and Remote Sensing Symposium,pages 2284-2287, July 2012.

[4] VPS Naidu. Multi-resolution image fusion by fft. In Image Information Processing (ICIIP), 2011 International Conference on, pages 1-6. IEEE,2011.

[5] F. Nencini, A. Garzelli, S. Baronti, and L. Alparone. Remote sensing image fusion using the curvelet transform. Information Fusion, 8(2):143-156, 2007.

[6] C. Pohl and J. L. Van Genderen. Review article multisensor image fusion in remote sensing: concepts, methods and applications. International journal of remote sensing, 19(5):823-854, 1998.

[7] Ante Poljicak, Lidija Mandic, and Darko Agic. Discrete fourier transform-based watermarking method with an optimal implementation radius. Journal of Electronic Imaging, 20(3):033008-033008, 2011.

[8] Tania Stathaki. Image fusion: algorithms and applications. Academic Press, 2011.

[9] B. Yang, Z. L Jing, and H. T. Zhao. Review of pixel-level image fusion. Journal of Shanghai Jiaotong University (Science), 15(1):6-12, 2010.

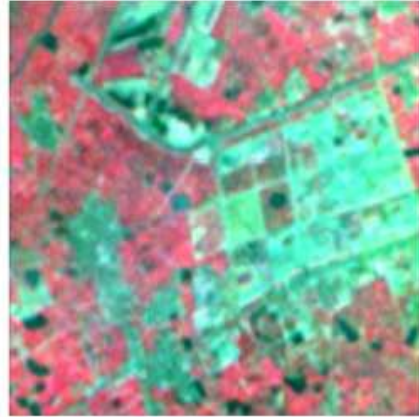

(a) MS

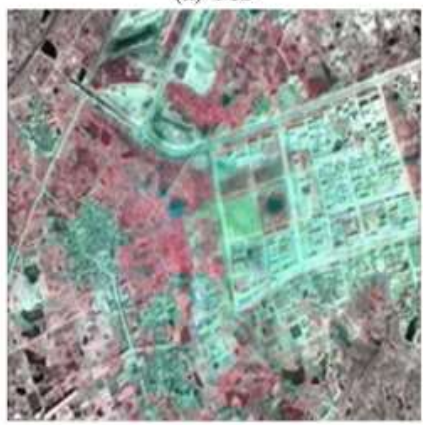

(c) FFT

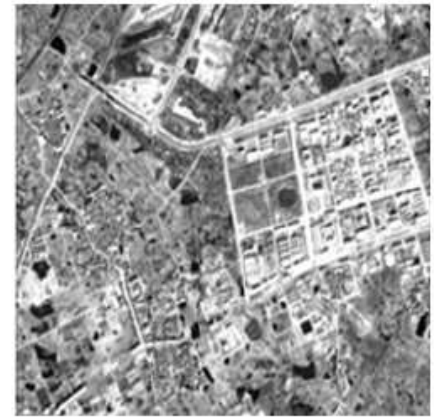

(b) PAN

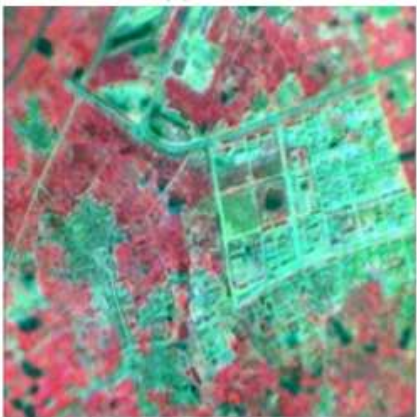

(d) FFT-HSV
Fig 3: Fusion of MS/PAN (Set 1) images

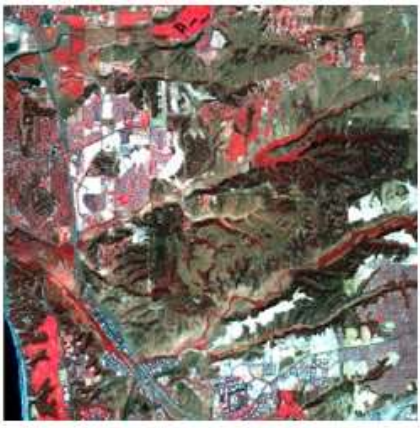

(a) MS

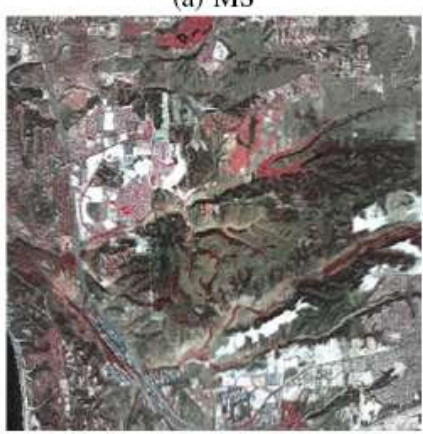

(c) FFT

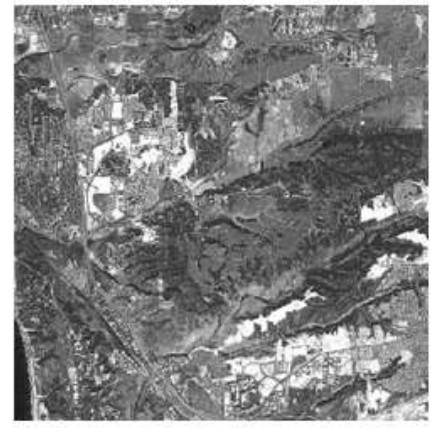

(b) PAN

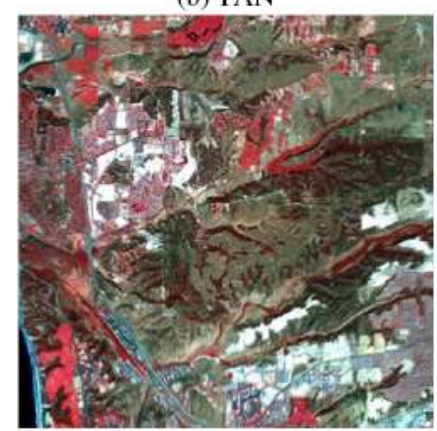

(d) FFT-HSV
Fig 4: Fusion of MS/PAN (Set 2) images 
International Journal of Trend in Scientific Research and Development, Volume 1(4), ISSN: 2456-6470 www.ijtsrd.com

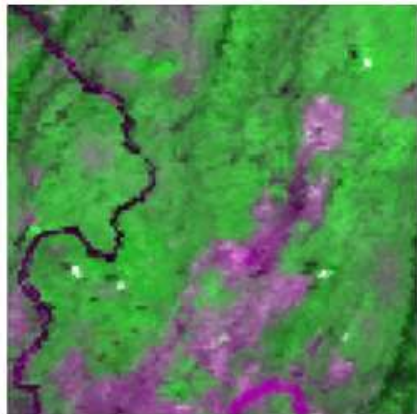

(a) MS

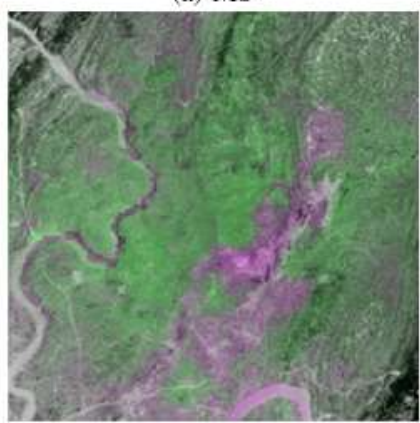

(c) FFT

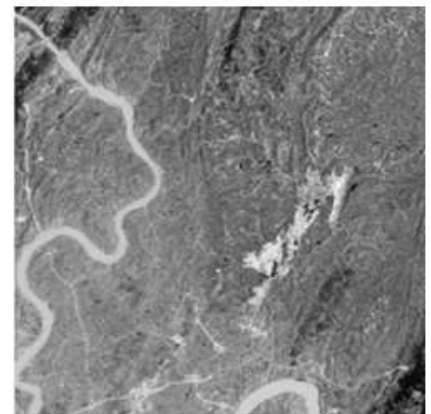

(b) PAN

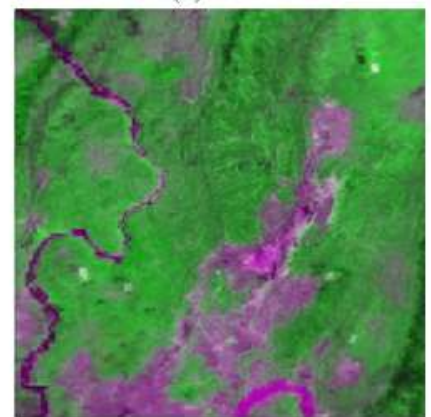

(d) FFT-HSV

Fig 5: Fusion of MS/PAN (Set 3) images 